\section{Clinical spectrum and surgical outcomes in spherophakia: a prospective interventional study}

S Khokhar, G Pillay, S Sen and E Agarwal

\begin{abstract}
Purpose To study the varied clinical presentations of patients with spherophakia, their management using surgical methods, and the clinical outcomes.

Patients and methods A prospective interventional study of 13 patients of spherophakia who presented to us from January 2014 and were followed up over the course of their treatment, and the data were documented for analysis.

Results In all, 26 eyes of 13 patients were reviewed and the median age of presentation was $12 \pm 12.05$ years. All patients had a bilateral presentation with 22 eyes having lenticular myopia with a mean refractive error of

$-11.5 \pm 12.945$ DS. Ten eyes presented with glaucoma of which six had raised intraocular pressure (IOP) $>21 \mathrm{~mm} \mathrm{Hg}$. A total of 23 eyes underwent lens extraction for dislocation/subluxation. Lens extraction helped lower overall IOP. Refractive rehabilitation was done with ACIOL, posterior chamber intraocular lens (PCIOL) with capsular tension ring, and scleral-

\section{Introduction}

Spherophakia is a rare congenital bilateral eye disorder, which presents with weak zonules around a smaller and more spherical crystalline lens with an increased anteroposterior thickness of the lens, and highly myopic eye. ${ }^{1}$ The lens zonules are developmentally hypoplastic and abnormally weak and due to non-attachment of the posterior zonules to the equatorial zone of the lens, the lens changes its normal shape to spherical. The lens may undergo subluxation or dislocation from the patellar fossa, leading to defective accommodation. The disease can present as an isolated condition or may run in families $^{2}$ and such cases have been reported in multiple lineage studies. Peculiarly in all these families, consanguinity seemed to be an important finding. ${ }^{1,3}$ Subluxation of lens may occur anteriorly, inferiorly, or posteriorly, ${ }^{4}$ and may lead to pupillary block glaucoma. ${ }^{5} \mathrm{We}$ hereby describe an interventional study of spherophakia patients who presented to our center with different manifestations of the disease and have been managed accordingly.
\end{abstract} fixated intraocular lens (SFIOL) in respective cases with ACIOLs being the most commonly used option.

Conclusions Spherophakia is a rare condition, which exhibits a varying degree of lenticular myopia, glaucoma, and subluxation of the crystalline lens. Lensectomy with proper rehabilitation using ACIOL, PCIOL, or SFIOL is a method of managing subluxation and unacceptable myopia. Lensectomy may also be a viable option of controlling glaucoma alongside medications and glaucoma surgery for the management of glaucoma in such cases.

Eye (2018) 32, 527-536; doi:10.1038/eye.2017.229; published online 3 November 2017

\section{Materials and methods}

This prospective interventional study evaluated the management strategies and outcomes of different presentations of microspherophakia cases, which were referred to our center. The study was done adhering to the tenets of the Declaration of Helsinki after taking proper approval from the Institutional Review Board of our hospital. Written informed consent was obtained from all included candidates. Included in this study were 13 patients of microspherophakia who presented to our center from January 2014 onwards and all included patients were followed up for a minimum period
Cataract and Refractive Surgery Services, Department of Ophthalmology, Dr. Rajendra Prasad Centre for Ophthalmic Sciences, All India Institute of Medical Sciences, New Delhi, India

Correspondence: G Pillay, Cataract and Refractive Surgery Services, Department of Ophthalmology, Dr. Rajendra Prasad Centre for Ophthalmic Sciences, All India Institute of Medical Sciences, Ayurvigyan Nagar, New Delhi 110049, India Tel: +91 9873028554; Fax: +91 02135232228. E-mail: ganesh.pillay@gmail. com

Received: 19 January 2017 Accepted in revised form:

17 August 2017

Published online:

3 November 2017 

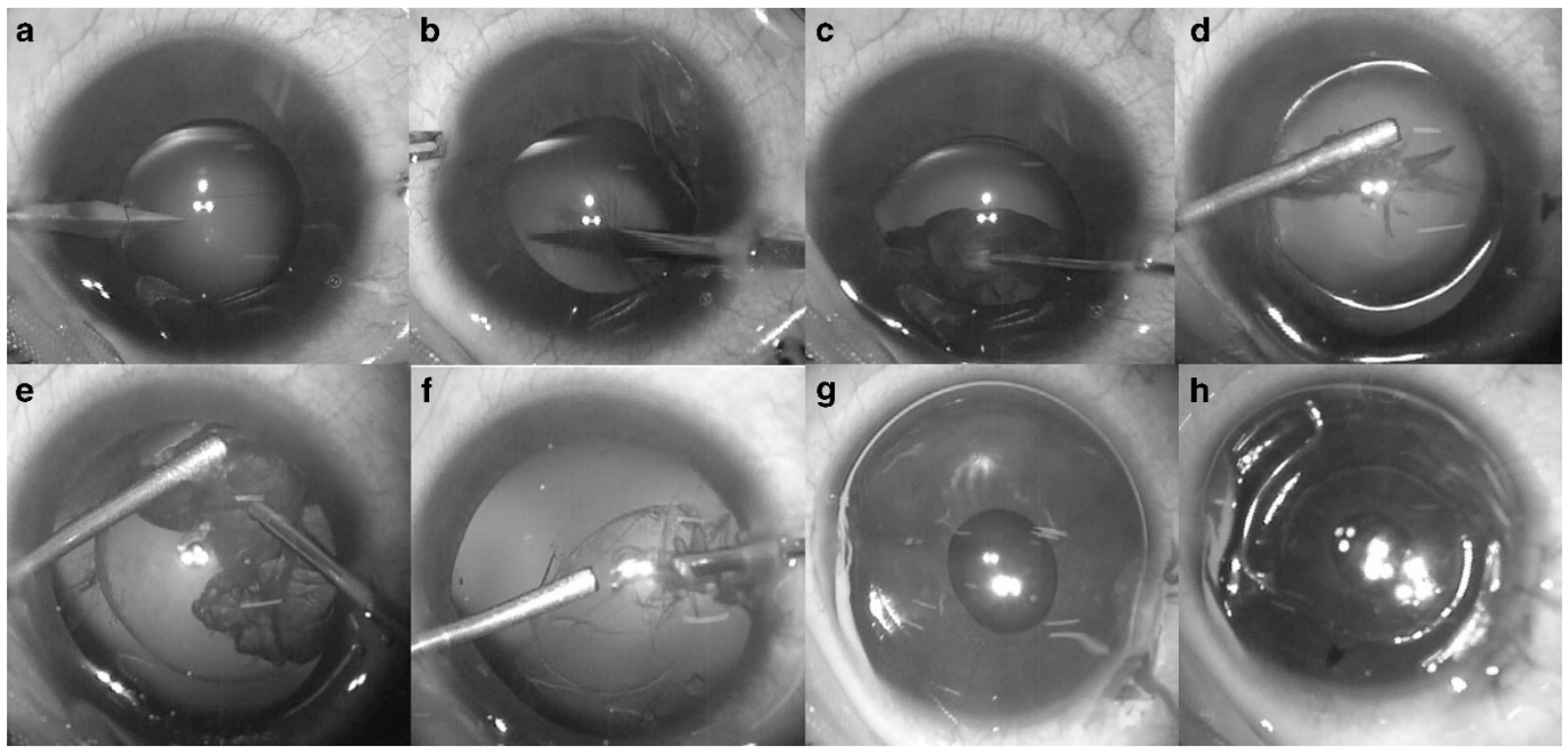

Figure 1 Endocapsular lens aspiration with insertion of ACIOL. (a) MVR entry is made in the cornea and extended to make a nick in the anterior capsule of the lens. (b) Two nicks are made in the anterior capsule with MVR blade. (c) Adrenaline is injected through one of the nicks for hydro dissection. (d) Irrigation cannula through opening and bag stabilized. (e) 25G vitrectomy cutter is inserted through one of the nicks and lens aspiration done in I-A mode. (f) Capsular bag is eaten and limited anterior vitrectomy is done with the cutter in cut I-A mode. (g) Pilocarpine followed by air is injected intracamerally and the iris is stroked with a Sinkey hook to constrict the pupil. (h) One of the corneal incisions is enlarged for the insertion of ACIOL and a PI is done. A full colour version of this figure is available at the Eye journal online.

of 1 year till November 2016. Data regarding demography, age at presentation, chief complaints, physical and ophthalmic examination, intraoperative findings, and postoperative examination were recorded, with a review of medical records and medication history. All patients were asked for family history status of a similar illness. All patients were evaluated for systemic status by a general physician/pediatrician.

Complete ophthalmic examination included preoperative best-corrected visual acuity (BCVA) using Snellen's chart at $6 \mathrm{~m}$, intraocular pressure (IOP) using non-contact applanation tonometer (NT-510, Nidek, Japan), slit lamp examination for anterior segment evaluation, dilated binocular indirect ophthalmoscopy for fundus, retinoscopy (phakic and aphakic) for refractive status, non-contact specular microscopy (SP 3000P, Topcon, Oakland, CA, USA) to determine the endothelial cell count (ECD), white-to-white (WTW) measurement using Orbscan II (Orbtek, Salt Lake City, UT, USA), central corneal thickness measurement with ultrasound pachymeter (IOPac Advanced, Heidelberg Engineering $\mathrm{GmbH}$, Germany), and biometry using IOLMaster 500 (Carl Zeiss Meditec, Jena, Germany). Patients with poor glow underwent a B-scan ultrasonography. Ultrasound biomicroscopy (Sonomed, Lake Success, NY, USA) with $50 \mathrm{~Hz}$ probe was performed for all cases to further delineate the anterior segment details. The patients also underwent screening for urine homocysteine levels and serum homocysteine levels were measured for those in whom urine screening came positive. Postoperatively, BCVA, IOP, refractive status, fundus, and endothelial counts were checked for all.

\section{Surgical techniques}

All surgeries were performed under sterile operation settings by the same surgeon (SK). General anesthesia was used for patients $<16$ years of age and peribulbar anesthesia for those above (Supplementary Video 1). Two clear corneal incisions were made using a 23G microvitreoretinal blade (MVR; Alcon Laboratories, Inc., Fort Worth, TX, USA) at 10 o'clock and $2 o^{\prime}$ clock positions with further insertion of the tip of the blade into the anterior capsule of the lens (Figure 1a and b). A viscodispersive agent (Viscoat; Alcon Laboratories, Inc.) was injected at the site of the zonular stretch to prevent vitreous entry into the anterior chamber. Vitrectomy cutter of Centurion system (25G, Alcon Laboratories, Inc.) was introduced through one incision and irrigation cannula through the other within the capsular bag and keeping the cutter in irrigation-aspiration cut (I-A cut) mode (Figure 1c and d), the lens matter was aspirated keeping vacuum at $400 \mathrm{~mm} \mathrm{Hg}$, aspiration flow rate at 50 c.c.s. / $\mathrm{min}$ and cut rate at 100 c.p.m. (Figure 1e). The capsular bag was then eaten keeping the cutter vacuum at $250 \mathrm{~mm} \mathrm{Hg}$, aspiration flow rate at 20 c.c.s. $/ \mathrm{min}$ and cut 
rate at 4000 c.p.m. and a limited anterior vitrectomy was performed in cut I-A mode to free the anterior chamber of vitreous strands (Figure 1f). An anterior chamber intraocular lens (IOL) was placed, after enlarging the main entry wound using a $5.2 \mathrm{~mm}$ single-bevel keratome (Alcon Laboratories, Inc.) (Figure $1 \mathrm{~g}$ and $\mathrm{h}$ ). The anterior chamber was formed by sterile air, and steroidsantibiotics combination was injected subconjunctivally. A peripheral iridotomy (PI) was made superiorly with the help of vitrectomy cutter in I-A cut mode in cases where ACIOL was implanted. The main corneal wound and the two side ports were sealed with stromal hydration using balanced salt solution and 10-0 nylon suture was placed if necessary. In cases where bag needed to be preserved, after making MVR entries, capsulorhexis was started from the 12 o'clock meridian with a 26-gauge cystitome under sodium hyaluronate 1.4\% (Healon GV, Pharmacia Upjohn, Kalamazoo, MI, USA) and finished with Utrata capsulorhexis forceps in the anticlockwise direction to avoid excessive zonular stretching. A modified capsular tension ring (M-CTR) was placed to stabilize the bag. Viscoelastic was removed from the anterior chamber with I-A probe. In all cases, a superior clear corneal incision was made with a $2.2 \mathrm{~mm}$ single-bevel keratome (Alcon Laboratories, Inc.) (not made in patients finally left aphakic). Healon 1.0\% (Pharmacia Upjohn) was injected to stabilize the anterior chamber and repeating the A-scan biometry under sterile conditions, a posterior chamber IOL was implanted if the bag was intact. In eyes with a posteriorly dislocated lens, a core vitrectomy with 25G+ vitrectomy cutter was performed followed by fragmentation of dropped cataractous spherophakia lens with fragmatome, with a scleral-fixated lens insertion and haptic burial under the scleral tunnel.

Postoperatively, the patients were advised topical prednisolone phosphate 1\% (P-lone, Syntho

Pharmaceuticals Pvt. Ltd., Lucknow, India) six times a day, moxifloxacin hydrochloride $0.5 \%$ three times a day (Vigamox, Alcon Laboratories, Inc.), and homatropine 2\% four times a day. Slit lamp examination was done postoperatively to assess the corneal clarity, the anterior chamber integrity, and the status of the IOL. IOP was measured using non-contact applanation tonometer. Specular microscopy was done at 1 month postoperatively to determine the endothelial cell status.

\section{Results}

Age of patients at presentation ranged from 2 to 40 years with a median age of $12 \pm 12.06$ years. Six patients were 10 years or younger and seven patients were older. All the patients were followed up for a minimum period of 1 year. Clinical details of the included patients have been tabulated in Table 1 along with the intraoperative and postoperative findings. The commonest presenting complaint was the diminution of vision $(n=24)$ followed by pain $(n=5)$ with 22 eyes having high myopia. Other patients were effectively aphakic due to the dislocation/ significant subluxation of the crystalline lens from the patellar fossa. All the examined patients had a bilateral presentation of the disease and 20 eyes presented with some form of subluxation of the spherical lens, either anteriorly, superonasally, or inferiorly. In all, 19 eyes had shallow or irregular anterior chamber with 8 eyes displaying iridodonesis and 6 eyes with frank phacodonesis. Six eyes presented with corneal edema, the most common cause being shallow anterior chamber and corneo-lenticular touch $(n=4)$ followed by raised IOP $(n=2)$.

Snellen's visual acuity was determined for 26 eyes and a BCVA of $>6 / 60$ was present in 13 eyes. The mean refractive error at presentation was $-11.5 \pm 12.45$ DS. Mean axial length of 24 eyes was $22.86 \pm 2.13 \mathrm{~mm}$ with mean WTW of $12.43 \pm 0.85 \mathrm{~mm}$ and mean keratometry value of $43.2 \pm 2.17 \mathrm{~mm}$. Six eyes had axial lengths of $>24 \mathrm{~mm}$. The ocular parameters have been tabulated in Table 2.

In all, 23 eyes were operated for lensectomy in view of the crystalline lens in the anterior chamber, an anteriorly dislocated lens with or without pupillary block glaucoma, inferior dislocation of the lens causing lenticular astigmatism, or posteriorly dislocated lens in the vitreous cavity. Refractive rehabilitation was done using anterior chamber IOLs in 13 eyes, posterior chamber IOL with CTR fixation in 3 eyes, and scleral-fixated glued IOL in 2 eyes. Seven eyes were left aphakic. BCVA after surgery was $>6 / 60$ in $18(78.3 \%)$ operated eyes with $>6 / 18$ in 10 $(43.5 \%)$ eyes, whereas preoperatively BCVA $>6 / 60$ was present in $13(56.5 \%)$ eyes and $>6 / 18$ in $4(17.4 \%)$ eyes. Visual acuity of case 8 with corneal decompensation did not improve after surgery due to persisting edema. BCVA of both eyes of case 7 improved to 6/9 although the acceptance with the previous correction of -32 DS OD and -33 DS OS was 6/18 in both eyes and this was attributable to minification factor of thick concave lenses.

\section{Management of glaucoma}

Five patients presented with secondary glaucoma. Two eyes of two patients presented with grade 2 corneal edema due to glaucoma. Six of the ten eyes presenting with glaucoma had IOP of $>21 \mathrm{~mm} \mathrm{Hg}$, which underwent lensectomy and the IOPs came down to $<16 \mathrm{~mm} \mathrm{Hg}$. Two of these eyes had the crystalline lens in the anterior chamber with the pupil constricted and open anterior chamber angles and the rest had pupillary block mechanism of glaucoma. In addition to lensectomy, these patients also received preoperative glaucoma medication. 


\begin{tabular}{|c|c|c|c|c|c|c|c|c|c|c|c|c|c|}
\hline 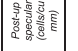 & 气̄ & & 票 & 总 & 兽 & $\overline{\bar{\sigma}}$ & $\Phi$ & $\approx$ & & 兽 & 甯 & $\frac{0}{5}$ & శ్ \\
\hline 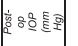 & $=$ & + & $\simeq$ & 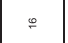 & - & + & $\simeq$ & 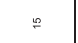 & $=$ & $\simeq$ & $\div$ & \pm & $=$ \\
\hline 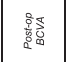 & 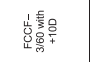 & & \% & 卷 & 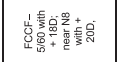 & 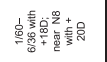 & 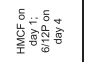 & 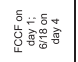 & & $\frac{\circ}{6}$ & 莡 & 产 & $\frac{\infty}{\bar{\omega}}$ \\
\hline $\bar{a}$ & g & w & 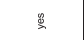 & 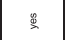 & 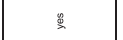 & 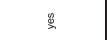 & $g$ & 黑 & g & 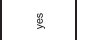 & 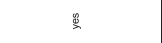 & ? & 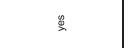 \\
\hline 高 & 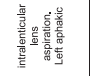 & & 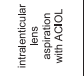 & 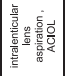 & 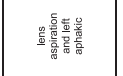 & 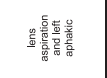 & 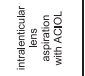 & 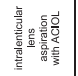 & & 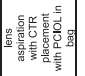 & 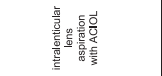 & 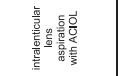 & 亭 \\
\hline 吡焉 & 器 & 泡 & : & $\overline{6}$ & 总 & 点 & $\approx$ & 总 & $\frac{9}{10}$ & 豆 & $\approx$ & : & ஜ \\
\hline 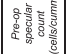 & 畄 & 釆 & 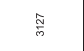 & 吉 & 蒚 & 槡 & 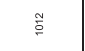 & 盒 & $\overline{\mathrm{s}}$ & 采 & ळ & 产 & 等 \\
\hline है & $\stackrel{\infty}{=}$ & $\stackrel{\circ}{=}$ & $\stackrel{\bullet}{=}$ & $\stackrel{?}{=}$ & 号 & $\nsubseteq$ & $\dddot{\varpi}$ & 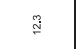 & $\dddot{\varpi}$ & $\stackrel{g}{\stackrel{g}{*}}$ & $\simeq$ & $\stackrel{?}{=}$ & $\cong$ \\
\hline$\frac{15}{2} 8$ 点 & 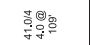 & 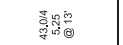 & 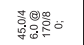 & 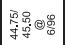 & 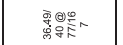 & 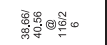 & 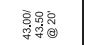 & 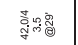 & 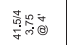 & 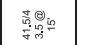 & 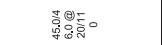 & 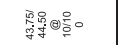 & 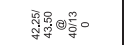 \\
\hline z & $\stackrel{\mathscr{d}}{q}$ & सं & 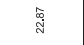 & $\overbrace{\text { ปn }}^{2}$ & $\stackrel{\sigma}{\bar{\sigma}}$ & $\stackrel{\mathscr{8}}{\sim}$ & $\frac{g}{2}$ & 茜 & $\overline{\bar{i}}$ & $\stackrel{g}{d}$ & ₹ & $\stackrel{:}{g}$ & 总 \\
\hline 高高 & 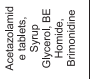 & & & & 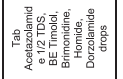 & & & & & & 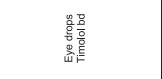 & 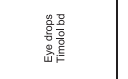 & \\
\hline 高高 & 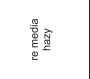 & 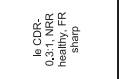 & 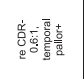 & 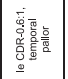 & 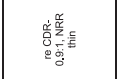 & 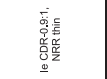 & 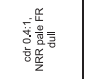 & 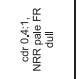 & 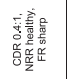 & 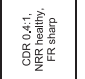 & 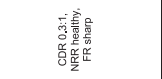 & 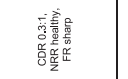 & 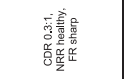 \\
\hline$\stackrel{2}{\xi}$ & 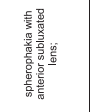 & & 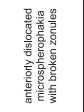 & 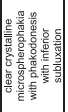 & 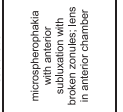 & 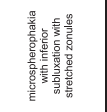 & 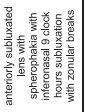 & 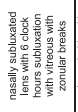 & & 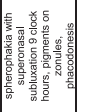 & 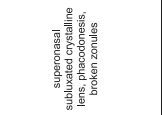 & 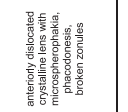 & 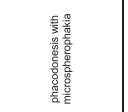 \\
\hline $8 \bar{\varepsilon}$ & $\stackrel{\bar{x}}{=}=$ & $\stackrel{\bar{g}}{g}=$ & 을 & 을 & 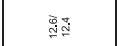 & 产西 & ฐ̃ & ฐ̃ & 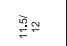 & 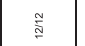 & 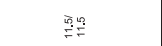 & E & 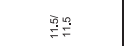 \\
\hline 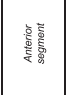 & 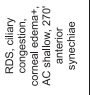 & 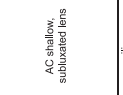 & 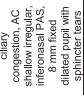 & 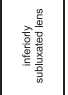 & 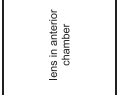 & & 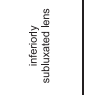 & 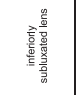 & 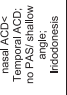 & 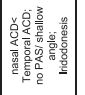 & 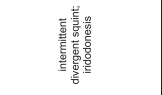 & 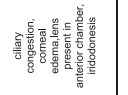 & 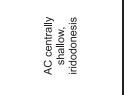 \\
\hline 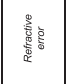 & 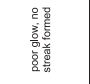 & 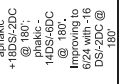 & 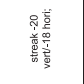 & 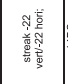 & 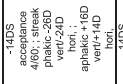 & 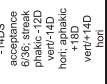 & 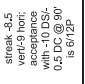 & 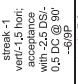 & 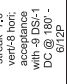 & 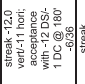 & 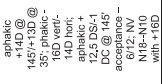 & 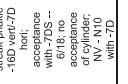 & 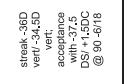 \\
\hline 謇 & 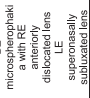 & & 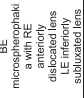 & & 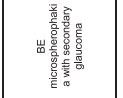 & & 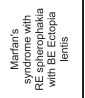 & & 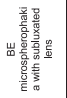 & & 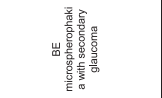 & & 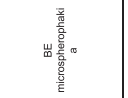 \\
\hline 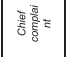 & 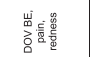 & & 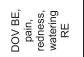 & & 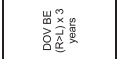 & & 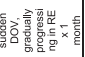 & & 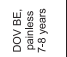 & & 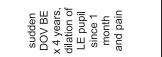 & & 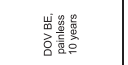 \\
\hline 商高 & 气ू & & 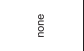 & & $\frac{\varrho}{\frac{g}{2}}$ & & 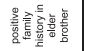 & & 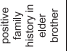 & & 气气 & & 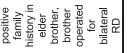 \\
\hline 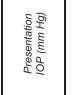 & 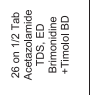 & 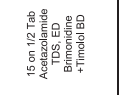 & 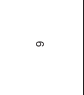 & $\nsubseteq$ & 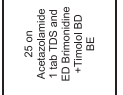 & 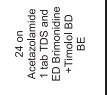 & $\infty$ & 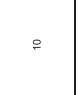 & \pm & $\simeq$ & $\approx$ & $\approx$ & \pm \\
\hline 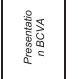 & 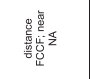 & 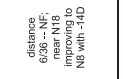 & 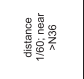 & 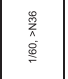 & 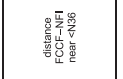 & 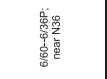 & 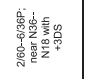 & 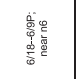 & 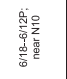 & 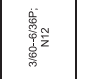 & 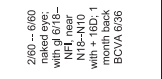 & 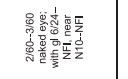 & 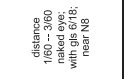 \\
\hline & 00 & on & 00 & 00 & 00 & $0 \infty$ & 00 & 00 & 00 & 000 & 00 & 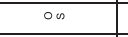 & 00 \\
\hline 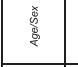 & 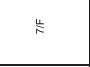 & & 產 & & 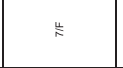 & & 㒳 & & 訔 & & 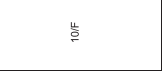 & & 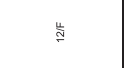 \\
\hline 象高高 & - & & " & - & $\circ$ & $\circ$ & - & $\infty$ & 。 & $\therefore$ & $=$ & $\simeq$ & $=$ \\
\hline 总 & \multicolumn{2}{|c|}{-} & \multicolumn{2}{|l|}{ N } & \multicolumn{2}{|c|}{$\infty$} & \multicolumn{2}{|c|}{+} & \multicolumn{2}{|c|}{$\infty$} & \multicolumn{2}{|l|}{ 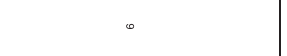 } & - \\
\hline
\end{tabular}




\begin{tabular}{|c|c|c|c|c|c|c|c|c|c|c|c|c|}
\hline$\frac{\mathrm{z}}{\mathrm{N}}$ & 今̆ & & $\frac{\mathscr{g}}{2}$ & æֶ & $\overline{\bar{s}}$ & $\frac{\mathrm{g}}{\mathrm{z}}$ & 弟 & 彎 & §ั & $\mid$ & $\frac{2}{N}$ & 喜 \\
\hline$\simeq$ & $\because$ & $\because$ & $=$ & 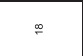 & $\infty$ & $\therefore$ & . & + & . & . & $\therefore$ & $\simeq$ \\
\hline \& & 譬 & & 荥茫 & 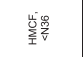 & 譥产 & 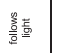 & $\frac{\varrho}{\varrho}$ & 总 & $\frac{\varrho}{\bar{\omega}}$ & $\stackrel{\frac{\Phi}{\tilde{\omega}}}{\bar{\omega}}$ & $\frac{\mathscr{O}}{\mathrm{w}}$ & $\frac{\mathrm{Y}}{\mathrm{\sigma}}$ \\
\hline 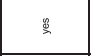 & 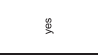 & 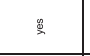 & 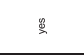 & 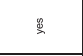 & 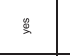 & 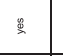 & $\approx$ & $\cong$ & 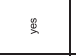 & 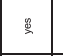 & 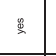 & 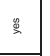 \\
\hline 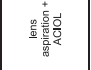 & 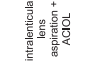 & & 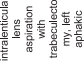 & 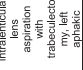 & 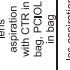 & 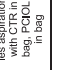 & 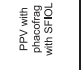 & 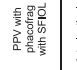 & 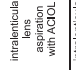 & 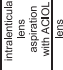 & 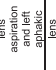 & 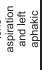 \\
\hline ฐ̊ & §̊ & $\overline{\bar{\omega}}$ & 吕 & : & $\mathrm{z}$ & $\overline{\bar{D}}$ & 楞 & 吕 & $\overline{\bar{s}}$ & $\hat{\mathrm{z}}$ & 总 & $\frac{\circ}{b}$ \\
\hline 踏 & $\frac{\mathrm{D}}{\mathrm{m}}$ & 急 & $\frac{\mathscr{2}}{\pi}$ & $\stackrel{ \pm}{\nexists ~}$ & ‡ & 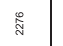 & 剱 & ฐ̊ & 袁 & 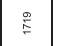 & $\stackrel{8}{2}$ & 㩊 \\
\hline 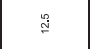 & 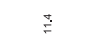 & $\stackrel{\infty}{=}$ & $\cong$ & $\underline{\underline{m}}$ & $\stackrel{2}{m}$ & $\underline{\underline{z}}$ & $\stackrel{\circledast}{\dddot{N}}$ & $\bar{I}$ & $\dddot{\dddot{I}}$ & $\cong$ & $\Xi$ & $\stackrel{\oplus}{=}$ \\
\hline 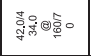 & 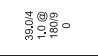 & 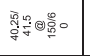 & 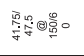 & 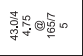 & 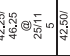 & 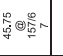 & 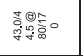 & 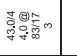 & 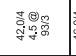 & 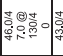 & 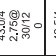 & 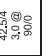 \\
\hline$\stackrel{g}{g}$ & 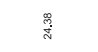 & สั & 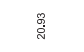 & 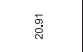 & $\stackrel{\Sigma}{\vdots}$ & $\stackrel{2}{\approx}$ & $\bar{\Sigma}$ & ֶָ & $\frac{\mathrm{g}}{\mathrm{N}}$ & $\frac{g}{\pi}$ & 亏 & $\frac{8}{8}$ \\
\hline & & & 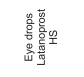 & 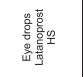 & & & & & 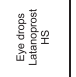 & 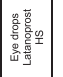 & & \\
\hline 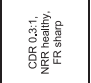 & 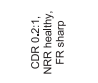 & 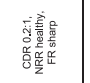 & 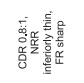 & 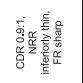 & 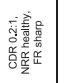 & 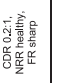 & 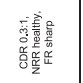 & 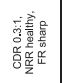 & 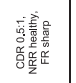 & $\mid$ & 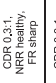 & 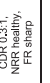 \\
\hline 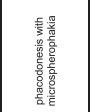 & 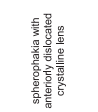 & 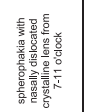 & 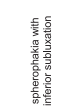 & 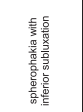 & 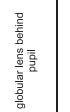 & 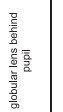 & 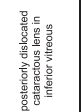 & 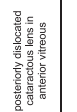 & 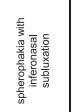 & 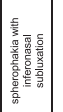 & 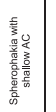 & 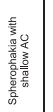 \\
\hline 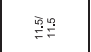 & 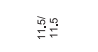 & $\stackrel{\Phi}{\cong} \cong$ & 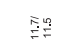 & 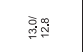 & 唁 & 旁 & 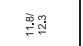 & 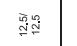 & W & פू & 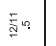 & 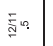 \\
\hline 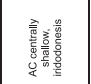 & 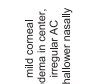 & 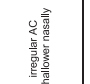 & 譥 & 嗙 & 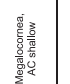 & 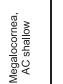 & 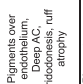 & 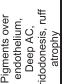 & 高 & 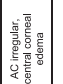 & 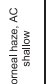 & 妥 \\
\hline 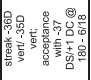 & 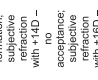 & 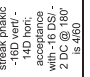 & 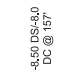 & 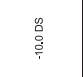 & 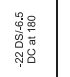 & 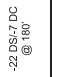 & 量: & 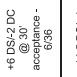 & 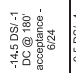 & 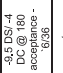 & 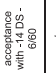 & 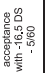 \\
\hline & 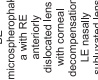 & & 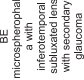 & & 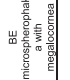 & & 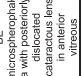 & & 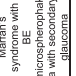 & & 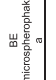 & \\
\hline & 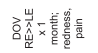 & & 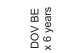 & & 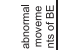 & & 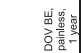 & & 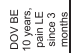 & & 嗐请 & \\
\hline & 旁 & & 彎 & & ڤ్ & & 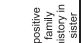 & & 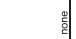 & & & \\
\hline 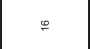 & $\because$ & $\simeq$ & $\cong$ & a & 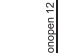 & 5 & & & & & & \\
\hline 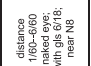 & 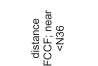 & 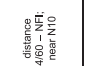 & 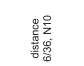 & 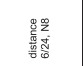 & 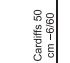 & 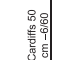 & 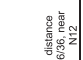 & 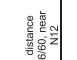 & & 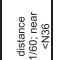 & 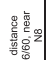 & 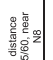 \\
\hline $0 \infty$ & 00 & on & 00 & on & 00 & ond & $\circ 0$ & on & 00 & on & 00 & on \\
\hline & 岑 & & 言 & & $\Sigma$ & & ثิ & & 촟 & & $=$ & \\
\hline \pm & $\because$ & $\because$ & $=$ & $\cong$ & 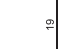 & & $=$ & $\approx$ & $\approx$ & 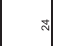 & 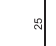 & $\approx$ \\
\hline & \multicolumn{2}{|c|}{. } & \multicolumn{2}{|c|}{ 。 } & \multicolumn{2}{|l|}{ 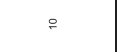 } & \multicolumn{2}{|l|}{$=$} & \multicolumn{2}{|l|}{$\cong$} & \multicolumn{2}{|c|}{$=$} \\
\hline
\end{tabular}


Table 2 Ocular parameters of sample population

\begin{tabular}{lcc}
\hline Clinical parameters & Mean $\pm S D$ & \\
\hline IOP at presentation $(n=26)$ & $15.46 \pm 4.89$ & $16.13 \pm 5.41$ \\
Preoperative IOP of operated eyes $(n=23)$ & $10.39 \pm 4.02$ & $P$-value $<0.001$ \\
Postoperative IOP of operated eyes $(n=23)$ & $2633.35 \pm 668.52$ & $P$-value $=0.36$ \\
Preoperative endothelial cell count of operated eyes $(n=21)$ & $2455.64 \pm 652.36$ & \\
Postoperative endothelial cell count of operated eyes $(n=21)$ & $544.19 \pm 27.09$ & \\
Preoperative CCT $(n=24)$ & & \\
\hline
\end{tabular}

Two patients had to be started on oral carbonic anhydrase inhibitors apart from topical multi-drug therapy and three patients could be managed with monotherapy. Nineteen eyes had normal optic nerve head $(\mathrm{ONH})$, whereas six eyes showed increased $\mathrm{ONH}$ cupping and glaucomatous changes with high IOP at presentation. Fundus for one eye could not be evaluated in view of corneal edema and media haze. Eyes that had an ACIOL implantation all underwent PI. Fellow eyes with spherophakia, which had been left for observation also underwent a PI. Two eyes of one patient (case 9) showed elevated pressures and advanced glaucomatous cupping at presentation for which a combined lensectomy with trabeculectomy was performed and on follow-up he continuously showed elevated pressures $(>40 \mathrm{~mm} \mathrm{Hg}$ ) for which bleb revision surgery had to be done in both eyes following which the IOPs came down to $<10 \mathrm{~mm} \mathrm{Hg}$. Case 3 presented with raised IOP $>30 \mathrm{~mm} \mathrm{Hg}$ in the right eye at 3 months' follow-up after lensectomy on multiple drug therapy and underwent trabeculectomy in right eye following which the IOP came down to $<10 \mathrm{~mm} \mathrm{Hg}$.

\section{Systemic associations}

Four patients (cases 4, 5, 7, and 11) had positive family history of spherophakia in their siblings (elder brothers in 4,5 , and 7, sister in 11), of whom two patients (cases 4 and 11) were known cases of Marfan's syndrome with arachnodactyly and high-arched palate. Both these cases had aortic root dilatation. One of these three elder brothers had a history of spherophakia with high myopia and bilateral retinal detachments for which 25G vitreoretinal surgery with silicone oil injection had been performed. One patient (case 2) had a history of seizures since 3 years of age with a right-sided hemiparesis and was found to have raised urine and serum homocysteine levels diagnostic of homocystinuria; however, no family history was found. This child was referred to the Paediatrics department for further management. The rest patients were believed to be having isolated spherophakia.

\section{No intervention}

No surgical intervention was performed on three eyes, of which one eye had angle closure due to pupillary block and was kept on glaucoma medication. PI was performed for all of these eyes.

\section{Discussion}

Most spherophakia patients present in adolescence or early adulthood and patients having systemic associations may present earlier, the median age in our study being 12 years. Most of our patients had a history of insidious onset diminution of vision and pain in some of the cases of secondary glaucoma, with diminution of vision being the commonest complaint. The condition may be isolated, familial, or associated with systemic disorders like homocysteinemia, Weil-Marchesani syndrome, Marfan's syndrome, Alport's syndrome, hyperlysinemia, megalocornea, spherophakia-secondary glaucoma, arrhythmogenic right ventricular dysplasia type 1 , and so on. ${ }^{2}$ The spherical lens may dislocate superotemporally, inferonasally, or inferiorly. The intraocular findings are a spherical lens, iridodonesis, and axial myopia, and the physical features depend on the syndrome associated, like arachnodactyly/brachydactyly, increased upper segment-lower segment ratios, tall/short stature, high-arched palate, joint stiffness, and congenital heart defects. Homocysteine levels may be raised in the urine and serum. Patients may also have musculoskeletal or metabolic diseases. But patients usually do not have buphthalmos, enlarged cornea, abnormal angle structures, or increased axial length associated, which differentiates the condition from primary congenital glaucoma. Although most of our cases were isolated spherophakia with inferior subluxation, we have described one case of homocystinuria with a history of seizures and hemiparesis and two cases of Marfan's syndrome. Cases of homocystinuria have been found to be associated with seizures and hemiparesis due to a hypercoagulable state. ${ }^{6}$ Cardiovascular associations are present in virtually most adults with Marfan's. ${ }^{7}$ Both of 
our cases had aortic root dilatation associated but ECG was normal in all of them.

\section{Lens extraction/lensectomy}

Lensectomy has been previously described as an option for managing the dislocated lens, ${ }^{8}$ following which visual rehabilitation becomes important, with probable options being aphakic spectacles, contact lenses, and IOLs. The choice of the IOL depends largely on the surgeon and patient factors. Angle-supported anterior chamber lenses (ACIOL; Figure 2c) and iris-enclavated lenses ${ }^{9}$ are commonly used options. Posterior chamber IOL (PCIOL) with/without CTRs/segments (CTS) ${ }^{10}$ and scleral-fixated IOL (SFIOL) ${ }^{11}$ have also been described in some isolated case reports. After IOL implantation patients need to be followed for long term to observe for amblyopia and occlusion therapy. Despite all efforts, amblyopia becomes difficult to manage, with compliance of glasses usage being poor in children, and ametropic amblyopia has been reported in $50 \%$ of patients with familial lens subluxation. ${ }^{12}$
Angle-supported ACIOLs have been reported to be associated with corneal endothelial cell loss, peripheral anterior synechiae (PAS) formation and glaucoma due to chronic anterior chamber irritation and modern users may have become skeptical of its use in younger population. ${ }^{13}$ Dehgan et $\mathrm{al}^{14}$ used angle-supported ACIOLs in most of their aphakic patients combined with a PI, with only few cases of displacement, IOL capture, and endothelial touch, which only reaffirms the safety of a properly sized ACIOL surgery done in experienced hands. ${ }^{15,16}$ The angle-supported ACIOL when planned rather than implanted in a complicated case has better results. Irissupported ACIOLs is also an option for hereditary lens subluxation cases because of larger WTW in Marfan's cases posing difficulty in choosing angle-supported IOL size. Iris-enclavated lenses may be placed anterior ${ }^{9}$ or posterior ${ }^{17}$ to the iris. But these iris claw lenses are hinging on a light sensitive mobile structure. Although ACIOL entry requires a large corneal incision which may later lead to astigmatism, it does have the advantage of better pupillary mobility compared to iris claw lens. (Supplementary Video 2)
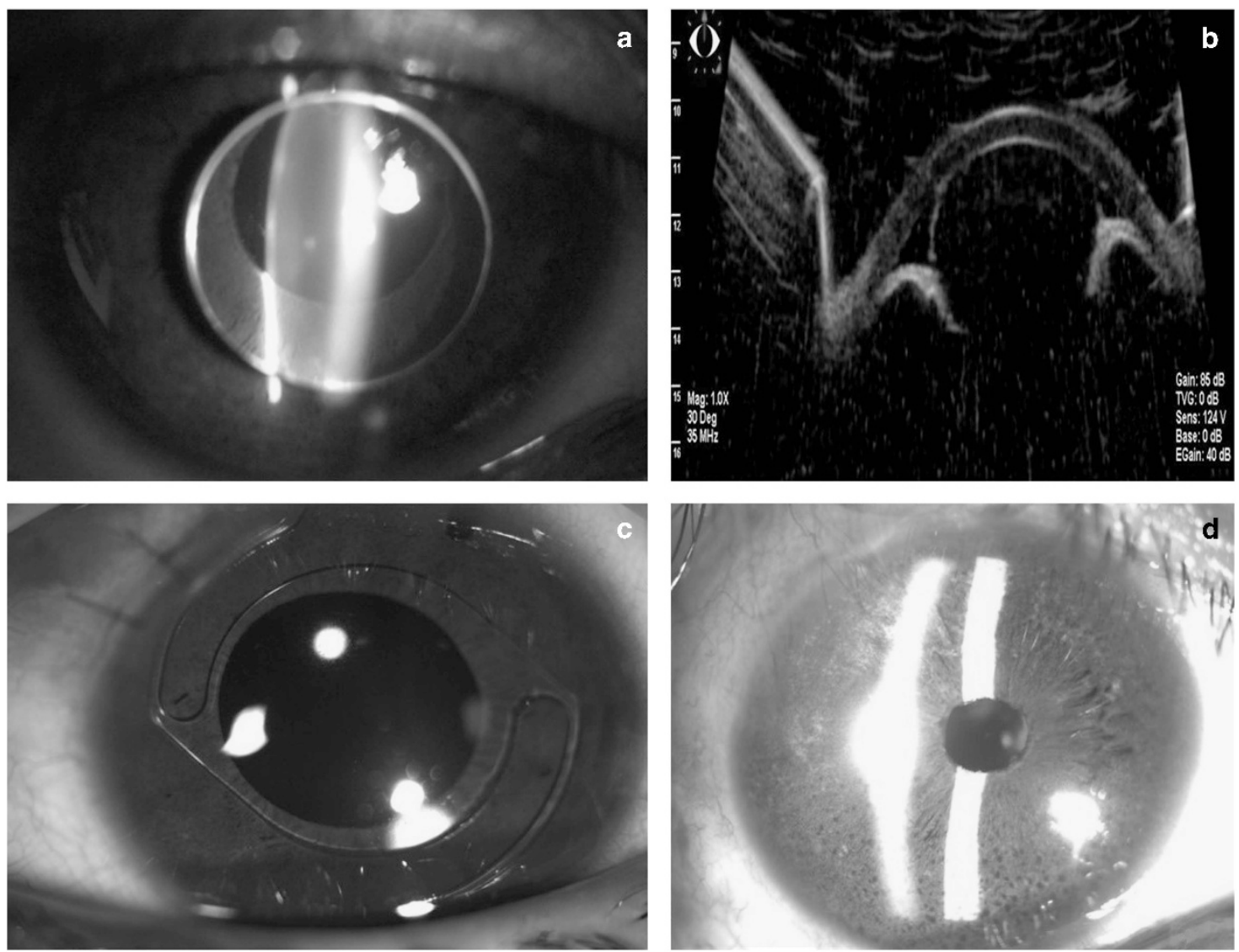

Figure 2 (a) Anteriorly dislocated spherophakic lens. (b) UBM showing lens in anterior chamber touching the cornea. (c) ACIOL in spherophakic patient. (d) SFIOL in spherophakic patient. A full colour version of this figure is available at the Eye journal online. 
Recently iris-supported ACIOLs have been used in pediatric aphakics with good visual outcome and no significant effect on the endothelium. ${ }^{18}$ Iris-supported ACIOLs seem to be a better option for hereditary lens subluxation cases because of larger WTW in Marfan's cases posing difficulty in choosing angle-supported IOL size. Iris-enclavated lenses may be placed anterior ${ }^{9}$ or posterior to the iris. Some studies have demonstrated that although immediate postoperatively ECDs may be affected, reported to be $1.5-10 \%$ at 3 years, ${ }^{19}$ they stabilize few years after. Although traumatic de-enclavations have been rarely reported till date, they are a cause for concern with these lenses ${ }^{20}$ specially in children. In spite of the popularity of Artisan iris-enclavated lenses, considering their inflated prices and easy local availability and affordability of angle-supported lenses, we opted for the latter in our study.

PCIOL placement is controversial as the zonules are developmentally weak and there is a possibility of the bag lens complex falling into the vitreous. ${ }^{21}$ Khokhar et al ${ }^{22}$ have described a 'dual-support technique' of insertion of a CTS with CTR in a case of spherophakia and have opined that this may help overcome zonular weakness. Also, Cionni (M-CTR) fixation to the sclera with 9-0 polypropylene (prolene) has been performed. A CTR has been said to strengthen the bag and reduce the risk of IOL subluxation.

SFIOL has been documented as a viable option for aphakic children (Figure 2d) and has been said to be rid of all these angle-related controversies, however, it requires an effective vitrectomy and proper scleral tunnel fixation. However, there has been a concern regarding SFIOL use in pediatric patients because of elastic and less rigid sclera compared to adults. SFIOL haptics have to be buried under the scleral flaps with polypropylene sutures and reports have shown that due to lack of fibrosis around lens loops, the suture is the only support for the lens. ${ }^{23}$ Transscleral suture exposure has been reported at 14.7$17.9 \% .^{23}$ Although prolene is theoretically nonabsorbable, there is a possibility of late decentration caused by suture degradation even years after, and this is particularly bothersome for the pediatric patient. ${ }^{24}$ Reports of suture breakage in SFIOLs further substantiate the concern. ${ }^{25}$ To summarize, SFIOL is a difficult procedure technically and may be associated with reported complications. ${ }^{13,26}$ In cases with a WTW $>13 \mathrm{~mm}$ such as megalocornea, SFIOL, or ACIOL are not preferred due to possible complications of IOL instability and decentration, respectively. Iris claw lens enclavated within the iris stroma may be more appropriate in such cases, with good visual outcome and no significant effect on the endothelium. ${ }^{18}$

None of our ACIOL patients presented with corneal decompensation. Likewise, PCIOL with M-CTR in the bag was stable and SFIOL patients did not present with IOL tilt or haptic extrusion till the last follow-up. The lenticular myopia of the order of -10 to $-15 \mathrm{D}$ is usually the first ophthalmologic finding in any spherophakia patient with usually normal axial lengths and corneal topography, ${ }^{27}$ which can considerably affect the quality of life. Hence, clear lens extraction with proper IOL placement can be considered as a method of correcting the unacceptably high myopia. In the study, visual improvement of $>6 / 18$ Snellen's was reported in $43.5 \%$ and $>6 / 60$ in $78 \%$ of our operated eyes. We believe that in this study we have reported the largest number of cases managed by lens extraction followed by different techniques of visual rehabilitation using mostly anglesupported ACIOL and few ones with PCIOL and SFIOL. The easy availability and affordability of angle-supported lenses makes it more viable compared to iris fixated and SFIOL specially in developing countries.

Spherophakia may be associated with angle-closure glaucoma, which may be because of pupillary block due to the spontaneous shift of iris-lens diaphragm anteriorly ${ }^{4}$ or the lens itself dislocating into the anterior chamber (Figure 2a) to cause angle block mechanically. The latter phenomenon has been labeled as inverse glaucoma ${ }^{28}$ and not unlike malignant glaucoma, this condition is deteriorated by miotics and relieved by mydriatic agents. ${ }^{29}$ Spontaneous dislocation has been described as a common cause of lens-induced glaucoma. ${ }^{30}$ Posttraumatic dislocation of the lens may also occur due to weak zonules. The migration of the lens into the anterior chamber may be intermittent causing acute glaucoma crises every time $\mathrm{e}^{5}$ and may eventually lead to retinal detachment due to the vitreous traction. A chronic pupillary block due to the forward migration of iris-lens diaphragm may result in crowding of the trabeculae (Figure 2b) and unrelieved, may lead to PAS formation and trabecular damage due to raised IOP. ${ }^{31}$ The lens may also dislocate posteriorly into the vitreous cavity ${ }^{4}$ as was found in one of our cases. Earlier concepts by Willi et al ${ }^{32}$ involved performing a laser PI to relieve pupillary block in such eyes and a surgical PI in case it failed. ${ }^{33}$ However, Asaoka et $a^{34}$ suggested that trabeculectomy was necessary to control the IOP. Another school of thought by Willoughby et $a l^{35}$ had developed, which stated that lensectomy would be able to control glaucoma in spherophakia, although Yasar ${ }^{36}$ suggested that it was a temporary option requiring a trabeculectomy subsequently for IOP control. Most of the eyes presenting with raised IOP in our study were managed with lensectomy with PI, which led to a significant lowered IOP $(P<0.001)$ in all of them and normal IOP on followup. One patient underwent combined lensectomy with glaucoma surgery followed by bleb revision, whereas another patient who presented with inverse glaucoma 
showed uncontrolled IOP post lens extraction and a trabeculectomy was performed for IOP control.

In summary, spherophakia is a disabling disease, which presents bilaterally with high myopia along with a risk of long-term glaucoma and systemic associations if it is a part of a syndromic manifestation. This can be tackled by lens extraction and IOL placement for visual rehabilitation and glaucoma treatment. Glaucoma management may be refractory and require further surgeries but overall the outcomes are good. The ACIOL are still a viable option when planned and put under experienced surgical hands.

\section{Summary}

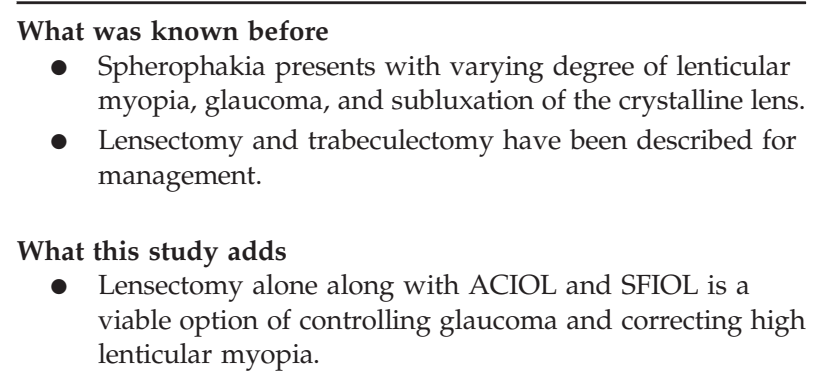

\section{Conflict of interest}

The authors declare no conflict of interest.

\section{References}

1 Ben Yahia S, Ouechtati F, Jelliti B, Nouira S, Chakroun S, Abdelhak $S$ et al. Clinical and genetic investigation of isolated microspherophakia in a consanguineous Tunisian family. J Hum Genet 2009; 54: 550-553.

2 Nelson LB, Maumenee IH. Ectopia lentis. Surv Ophthlamol 1982; 27: 143-160.

3 Kumar A, Duvvari MR, Prabhakaran VC, Shetty JS, Murthy GJ, Blanton SH. A homozygous mutation in LTBP2 causes isolated microspherophakia. Hum Genet 2010; 128: 365-371.

4 Desir J, Sznajer Y, Depasse F, Roulez F, Schrooyen M, Meire $\mathrm{F}$ et al. LTBP2 null mutations in an autosomal recessive ocular syndrome with megalocornea, spherophakia, and secondary glaucoma. Europ. J Hum Genet 2010; 18: 761-767.

5 Khan AO, Aldahmesh MA, Alkuraya FS. Congenital megalocornea with zonular weakness and childhood lensrelated secondary glaucoma-a distinct phenotype caused by recessive LTBP2 mutations. Mol Vis 2011; 17: 2570-2579.

6 Eslamiyeh H, Ashrafzadeh F, Akhondian J, Beiraghi Toosi M. Homocystinuria: a rare disorder presenting as cerebral sinovenous thrombosis. Iran J Child Neurol 2015; 9(2): 53-57.

7 Stuart AG, Williams A. Marfan's syndrome and the heart. Arch Dis Child 2007; 92(4): 351-356.

8 Muralidhar R, Ankush K, Vijayalakshmi P, George VP. Visual outcome and incidence of glaucoma in patients with microspherophakia. Eye 2015; 29: 350-355.
9 Lifshitz T, Levy J, Klemperer I. Artisan aphakic intraocular lens in children with subluxated crystalline lenses. J Cataract Refract Surg 2004; 30(9): 1977-1981.

10 Bhattacharjee $\mathrm{H}$, Bhattacharjee K. Clear lens extraction and intraocular lens implantation in a case of microspherophakia with secondary angle closure glaucoma. Indian J Ophthalmol 2010; 58(1): 67-70.

11 Subbiah S, Thomas PA, Jesudasan CA. Scleral-fixated intraocular lens implantation in microspherophakia. Indian J Ophthalmol 2014; 62(5): 596-600.

12 Romano PE, Kerr NC, Hope GM. Bilateral ametropic functional amblyopia in genetic ectopia lentis: its relation to the amount of subluxation, an indicator for early surgical management. Binocul Vis Strabismus Q 2002; 17(3): 235-241.

13 McAllister AS, Hirst LW. Visual outcomes and complications of scleral-fixated posterior chamber intraocular lenses. J Cataract Refract Surg 2011; 37: 1263-1269.

14 Dehghan M-H, Soleimanizad R, Ahmadieh H, Azarmina M, Soheilian M, Moradian S et al. Outcomes of lensectomy in hereditary lens subluxation. Iranian J Ophthalmic Res 2006; 1 (2): 92-95.

15 Wagoner MD, Cox TA, Ariyasu RG, Jacobs DS, Karp CL. Intraocular lens implantation in the absence of capsular support; a report by the American Academy of Ophthalmology (Ophthalmic Technology Assessment). Ophthalmology 2003; 110: 840-859.

16 Morrison D, Sternberg P Jr, Donahue S. Anterior chamber intraocular lens (ACIOL) placement after pars plana lensectomy in pediatric Marfan syndrome. JAAPOS 2005; 9: 240-242.

17 Dureau P, De Laage de Meux P, Edelson C, Caputo G. Iris fixation of foldable intraocular lenses for ectopia lentis in children. J Cataract Refract Surg 2006; 32: 1109-1114.

18 Sminia ML, Odenthal MT, Prick LJ, Cobben JM, Mourits MP, Völker-Dieben HJ. Long-term follow-up after bilateral Artisan aphakia intraocular lens implantation in two children with Marfan syndrome. J AAPOS 2012; 16(1): 92-94.

19 Qasem Q, Kirwan C, O'Keefe M. 5-year prospective followup of Artisan phakic intraocular lenses for the correction of myopia, hyperopia and astigmatism. Ophthalmologica 2010; 224: 283.

20 De Silva SR, Arun K, Anandan M, Glover N, Patel CK, Rosen $P$. Iris-claw intraocular lenses to correct aphakia in the absence of capsule support. J Cataract Refract Surg 2011; 37 (9): 1667-1672.

21 Khokhar S, Pangtey MS, Sony P, Panda A. Phacoemulsification in a case of microspherophakia. J Cataract Refract Surg 2003; 29: 845-847.

22 Khokhar S, Gupta S, Kumar G, Rowe N. Capsular tension segment in a case of microspherophakia. Cont Lens Anterior Eye 2012; 35: 230-232.

23 Lubniewski AJ, Holland EJ, Woodford S. Histologic study of eyes with transsclerally sutured posterior chamber intraocular lenses. Am J Ophthalmol 1990; 110: 237-243.

24 Price MO, Price Jr FW, Werner L, Berlie C, Mamalis N. Late dislocation of scleral-sutured posterior chamber intraocular lenses. J Cataract Refract Surg 2005; 31: 1320-1326.

25 Moreno-Montañés J, Fernández-Hortelano A, Caire J. Entrapment of a Cionni ring hook by the pupil after suture rupture. J Cataract Refract Surg 2008; 34: 1047-1048.

26 Rajpa RK, Carney MD, Weinberg RS, Guerry RK, Combs JL. Complications of transscleral sutured posterior chamber intraocular lenses. Ophthalmology 1991; 98(Suppl): S144. 
27 Johnson VP, Grayson M, Christian JC. Dominant microspherophakia. Arch Ophthalmol 1971; 85: 534-542.

28 Urbanek J. Glaucoma juvenile inversum. Z Augenheilkd 1930; 71: 171-172.

29 Willi M, Kut L, Cotlier E. Pupillary-block glaucoma in the Marchesani syndrome. Arch Ophthalmol 1973; 90: 504-508.

30 Jovanović M, Stefanović I. Spontaneous dislocation of a transparent lens to the anterior chamber-a case report. Srp Arh Celok Lek 2010; 138(7-8): 486-488.

31 Johnson GJ, Bosanquet RC. Spherophakia in a NewFoundland family: 8-year experience. Can J Ophthalmol 1983; 18: 159-164.

32 Willi M, Kut L, Cotlier E. Pupillary block glaucoma in the Marchesani syndrome. Arch Ophthalmol 1973; 90: 504-508.
33 Ritch R, Wand M. Treatment of the Weil Marchesani syndrome. Ann Ophthalmol 1981; 13: 665-667.

34 Asaoka R, Kato M, Suami M, Usami Y, Hotta Y, Sato M. Chronic angle closure glaucoma secondary to frail zonular fibres and spherophakia. Acta Ophthalmol Scand 2003; 81: 533-535.

35 Willoughby CE, Wishart PK. Lensectomy in the management of glaucoma in spherophakia. J Cataract Refract Surg 2002; 28(6): 1061-1064.

36 Yasar T. Lensectomy in the management of glaucoma in spherophakia: is it enough? J Cataract Refract Surg 2003; 29: 1052-1053.

Supplementary Information accompanies this paper on Eye website (http://www.nature.com/eye) 